\title{
Instantaneous Rock Blasting Wave and Its Microscopic Characteristics during Interaction with Concrete
}

\author{
Shaoqin Huang, ${ }^{1}$ Lifeng Luan, ${ }^{1}$ Wanli Xing, ${ }^{2}$ and Qunyi Liu ${ }^{2}$ \\ ${ }^{1}$ School of Earth Sciences and Resources, China University of Geosciences, Beijing 100083, China \\ ${ }^{2}$ Institute of Mineral Resources, Chinese Academy of Geological Sciences, Beijing 100037, China \\ Correspondence should be addressed to Wanli Xing; xingwanlicsgs@126.com
}

Received 30 September 2014; Revised 7 January 2015; Accepted 7 January 2015

Academic Editor: Shimin Liu

Copyright (c) 2015 Shaoqin Huang et al. This is an open access article distributed under the Creative Commons Attribution License, which permits unrestricted use, distribution, and reproduction in any medium, provided the original work is properly cited.

\begin{abstract}
This paper uses improved technology for dynamic strain measurement to investigate the dynamic strain signals of blasting wave action tested in the range of $8-16 \mathrm{~cm}$ from the central blast. Based on the blasting mechanism and on the analysis of signal characteristics, blasting waves are recognized and divided into three zones, namely, shock wave zone, stress wave zone, and gasexpanding zone. This paper studies the relationships between stress, strain, and time of every zone. The tensile and compressive stresses of stress wave are considered. After the blasting test, four cracks from the borehole center toward the minimum burden appear at the model surface. The relationship between blasting wave and concrete damage is analyzed.
\end{abstract}

\section{Introduction}

Concrete is often used to reinforce mine tunnels. In a mining excavation, the blasting excavation method is often applied. This method may damage the concrete reinforcement and consequently change the stress-strain response of concrete under static load. According to the mechanism of rock blasting, the combined effects of blasting shock wave, stress wave, and gas expansion (collectively called blasting wave in this paper) will be exerted on the area adjacent to the contact structure at the instant of blasting $[1,2]$. These waves are distinct from one another in terms of action time, action area, characteristics of loading and unloading, time-frequency distributions, allocation of energy, attenuation, and contribution to damage. Studies on the essential characteristics of blasting waves based on real-time tests are significant in many fields, such as mining engineering, as well as studies on stress-strain relations of rock mass [3-5], the characteristics of the source of blasting seismic wave $[2,6]$, and so on.

Thus far, some studies have investigated blasting waves at the instant of detonation or blasting [7-9]. Wen et al. [10] obtained the peak values of the shock wave between 0.81 and $22.3 \mathrm{GPa}$ in different positions. These references mainly investigated the detonations or the shock wave in the medium, thereby promoting understanding of the blasting mechanism. However, because of the complexity of blasting, the limitations of equipment to experiment on the adjacent area, and the constraints in technology, among others, only a few studies have investigated the tension phase after shock wave $[11,12]$. Few studies have made progress in the study of the combined effects of blasting shock wave, stress wave, and gas expansion on the adjacent area at the instant of blasting; the separation of each wave from the result; and the essential characteristics of these waves because of the difficulty in obtaining the blasting wave signals.

Based on laboratory tests, the transient blasting wave strain signals in the concrete model are tested using resistance strain gauges with a highly dynamic strain testing system. This study also quantitatively examines the blasting wave and the essential characteristics of the separated blasting shock wave, stress wave, and gas expansion, considering such factors as action time, action area, dynamic strain variation, characteristics of loading and unloading, velocity and acceleration of test points, and features of time-frequency energy.

\section{Blasting Test Results}

The specimens are precast concrete with size of $500 \mathrm{~mm} \times$ $500 \mathrm{~mm} \times 350 \mathrm{~mm}$ cuboid, as shown in Figure 1, at the center 


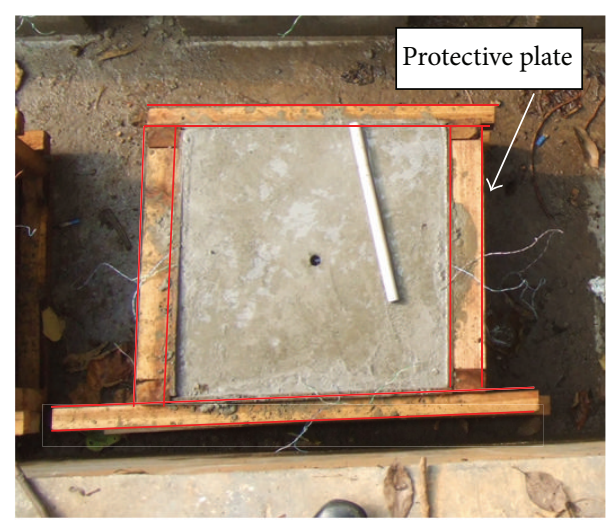

Figure 1: Concrete test model.

of which there is a borehole $250 \mathrm{~mm}$ deep and $8 \mathrm{~mm}$ in diameter. Six radial strain gauges are preburied in each model, and the test points (1) and (2) are $9 \mathrm{~cm}$ deep located along the direction of the minimum free face, 8 and $16 \mathrm{~cm}$ away from the center line of the borehole, respectively. To eliminate the influence of the free face surrounding the model, which may affect the blasting result, butter is coated onto the protective plate to constrict concrete block during the blasting.

A total of 108 test points of 18 models are tested for dynamic strain wave in this paper. The relationship between voltage signal $U_{0}$ and dynamic strain $\varepsilon_{d}$ is as follows:

$$
\varepsilon_{d}=\frac{4 U_{0}}{K U_{1} A},
$$

where $U_{0}$ is the output voltage $(V), U_{1}$ is the bridge voltage $(V), K$ is the sensitivity coefficient of the strain gauge, $\varepsilon_{d}$ is the dynamic strain of the strain gauge, and $A$ is the gain.

Assuming the specimens are in the elastic stage, the strain value can be obtained by $\sigma=E \varepsilon_{d}$. The time-strain curves of the test points (1) and (2), which are $8 \mathrm{~cm}$ and $16 \mathrm{~cm}$ away from the center line of the borehole, respectively, in different conditions, are shown in Figure 2 after being transformed by formula (1). Based on the test results, other blasting waves obtained in the test have the same forms as in Figure 2, indicating that the waveform in Figure 2 is universal and representative.

\section{Relation of the Division and the Detonation Structure}

The blasting wave division in concrete is fairly related to the detonation wave structure. According to ZND detonation theory [13-15], the spread of the shock wave in the dynamite will become the detonation wave. Additionally, the detonation wave structure can be divided into three parts: shock front compressive zone $0-1$, reaction zone $2-2$, and gasexpanding zone.

(1) The shock front compressive zone is about $10^{-4} \mathrm{~mm}$ wide and has the highest pressure and density, where values suddenly surge.

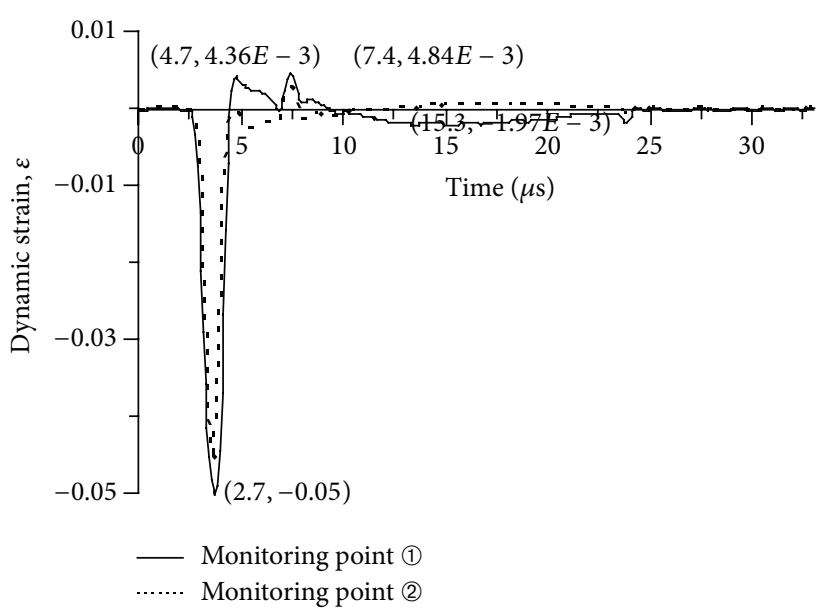

Figure 2: Complete time-strain curves of blasting waves.

(2) The reaction zone is about $10^{-1} \mathrm{~mm}$ wide. The action time is in the order of magnitude of $10^{-1} \mu \mathrm{s}$. The head face pressure $P_{H}$ (i.e., pressure of C-J plane) at 2-2 is about $1 / 2$ of the wave front pressure $P_{Z}$ at $0-1$, and the density at $2-2$ is about $1 / 3$ of the density at $0-1$.

(3) In the gas-expanding zone, the expansion of the detonation products is isentropic, and the air pressure, density, and temperature continue to fall.

As pointed out by Ramulu et al. [16], when the blasting occurs in the concrete, the effect of blasting wave on the concrete is similar to the detonation wave in an explosive and depends on the detonation design parameters. As proven by experiments, the shock wave in rocks is a disturbance at supersonic speed. The medium states (pressure, density, and temperature) at the wave front mutate in fractions of $1 \mu \mathrm{s}$, and the particles at the wave front move fast. The result agrees well with those shown in Figure 2, which means that the blasting shock wave in the concrete is the result of the sudden jump in detonation pressure at the wave front compressive zone. However, because the blasting shock wave spreads in the nonexplosion medium, the flowing velocity of the particles right behind the shock front is lower than the spreading velocity of the disturbance. Furthermore, because the shock wave lacks external support, some rarefaction waves exist, leading to an aperiodic decay of each parameter after the shock front in the concrete at 3-4 $\mu$ s. Therefore, the shock wave turns into the stress wave. The stress wave amplitude is much smaller than the shock wave amplitude. The stress wave front is also flatter, its action time equals that of the shock wave, and the time of the stress value ascending lasts shorter than when the stress value is descending. The stress wave zone in the concrete may be related to the continuous falling of the pressure and the density between the 1-1 front plane and the 2-2 (C-J) plane in the reaction zone. A certain concrete point in the stress zone may also correspond with a point at the C-J plane in the detonation wave. The gasexpanding zone in the concrete is caused by the amorphous isentropic expansion and flow of the detonation product after the C-J plane. And the gas-expanding action mainly causes 
the deformation, compression, and tension in the rocks, as well as the formation of cracks.

\section{Relation of the Wave Division and the Mechanism of Rock Blasting}

To summarize various perspectives on the mechanism of rock blasting, Yang et al. [17] considered that the stress wave plays a dominant role in the whole rock blasting process. In terms of time, the shock wave activates the original microcracks in rocks and generates the crushed zone and radial cracks around the borehole first; then the following stress wave widens these cracks, which leads to a reflected tension stress wave failure at the free face; and the explosion gas wedges in the cracks may connects the cracks together. Finally, the rock fragments are thrown out. Owing to the separation of the free face reflected wave and the blasting wave in the temporal domain, the gas-expanding zone right after the shock wave zone being the reflected tension stress wave is impossible, as proven by the measured reflected tension stress wave from the free face. The wave division in this test agrees well with the result of the study by Yang et al. [17], who supposed that the reflected tension stress wave from the free face is negligible.

In terms of space, when a coupling explosion occurs in an infinite rock, three zones will appear around the borehole. These zones are the crushed zone (near area), which is 3$7 R_{0}\left(R_{0}\right.$ is the charge radius) radius around the borehole and caused by the shock wave; the crack zone (middle area), which is $8-150 R_{0}$ radius around the borehole and caused by the stress wave; and the elastic vibration zone (far area), which is more than $150 R_{0}$ radius around the borehole and caused by the gas expansion. In this test, the monitoring points are located in the blasting center area at $20 R_{0}, 31 R_{0}$, and $40 R_{0}$ away from the center. The test results demonstrate that the blasting shock wave action still affects this area, and the fissures in the rocks at the center area are caused by the combined effects of blasting shock wave, stress wave, and gas expansion.

Therefore, the effects of coupling explosive blasting waves in the concrete can be divided into three phases in terms of time.

(1) The shock wave from the blasting wave front affects the concrete medium by the overpressure and violent shock at a loading or unloading strain rate of more than $10^{4} / \mathrm{s}$, which can be drawn from the phenomenon that at 2.4-3.7 $\mu$ s the concrete is under loading compression, at 2.4-3.7 $\mu$ s the blasting cavity is under unloading and resilience, and at $4.7-5.6 \mu \mathrm{s}$ the remaining weak shock wave is decaying with oscillation.

(2) The stress wave affects the concrete medium through the compression and tension stress at a loading or unloading strain rate several times higher than $10^{4} / \mathrm{s}$, which can be drawn from the phenomenon that the concrete is under loading and unloading again at 5.6$8.6 \mu \mathrm{s}$, and the peak values of the compression stress, the tension stress, and the strain rate at the broken boundary are much higher than before.

(3) The isentropic expansion of gas affects the medium by continuous approximate static tension or compression stress, which can be drawn from the phenomenon that at 8.6-24.7 $\mu$ s the concrete is still under continuous tension and compression, and decaying with oscillation occurs in the end lasting for about $1 \mu \mathrm{s}$.

In terms of space, these three actions exist in the study area at the same time, and they are independent of one another, contrary to the traditional view. The shock wave is previously thought to be in the near area, and the stress wave that decays rapidly with distance is in the middle area of the rock blasting area division. These two mechanical processes are two blasting loading forms in the medium because of their different distances from the blasting center, instead of being independent of each other.

\section{Verification of Blasting Wave Division}

To further verify the blasting wave division, the reflected blasting wave and the contacting blasting wave from the free face are tested on the same type of concrete. In the free face reflected wave, the protective plate is uncovered to prevent absorbing the energy. The sampling precision is $0.1 \mu \mathrm{s}$, and the sampling time lasts $20000 \mu \mathrm{s}$; thus, 200000 data sets of each point are recorded to form a blasting waveform during each blasting. A part of the original measured waveform can be seen in Figure 3, and other analogous waveforms during the whole test appeared. In Figure 3, two conspicuous sudden jumps are recorded. A zoomed in image of the waveform of jump (1) is shown in Figure 2. The waveforms of the two monitoring points of jump (2) are found in Figure 3(a) and zoomed in in Figure 4. The time in Figure 4 starts from the $4025 \mu \mathrm{s}$ in Figure 3(a), and the action time of the reflected wave is about $25 \mu \mathrm{s}$. As shown in the test result, the reflected wave cannot follow right after the shock wave zone and the stress wave zone. Additionally, the properties of the reflected waveform are entirely distinct from those of the waveform in the gas-expanding zone. Besides, no reference has ever proven that the free face reflected wave follows right after the blasting shock wave.

In the free face contacting blasting test, the charge is moved to the free face along the least resistance line, and everything is kept constant except for the blasting position. The measured waveform and the variations in loading strain rate with time in every contacting blasting can be found in Figure 5.

Based on Figure 5, the third zone of the blasting wave signal obviously disappears as the energy dissipates because of the rapid expansion in the air of the explosion gas. This expansion leads to a decrease in stress at the interface, such that the dynamic strain of the point in the medium is not high enough to be measured. If the blasting reflected wave is the one measured in the zone, the zone will not disappear because the free face stays and the reflected wave continues; the second zone, which is the stress wave zone, obviously 


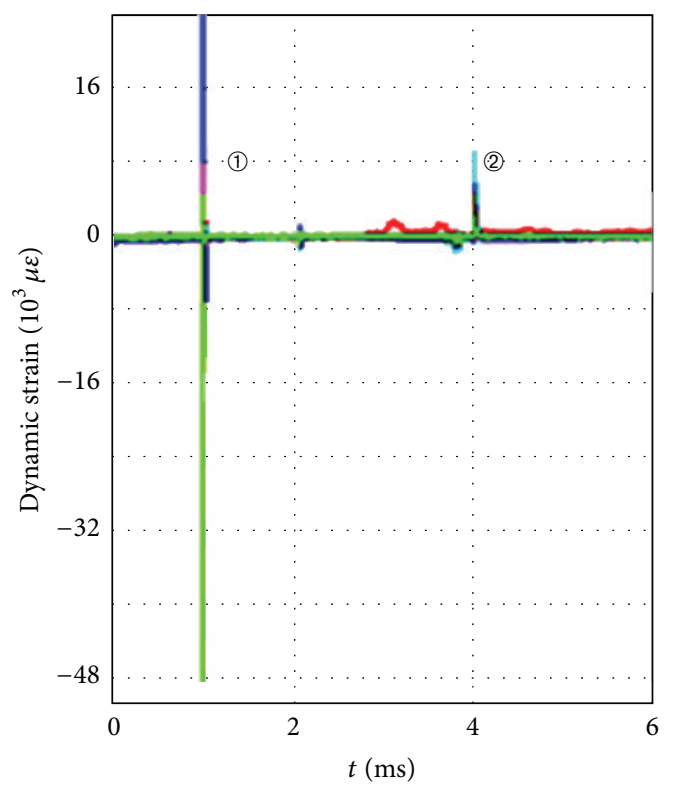

(a)

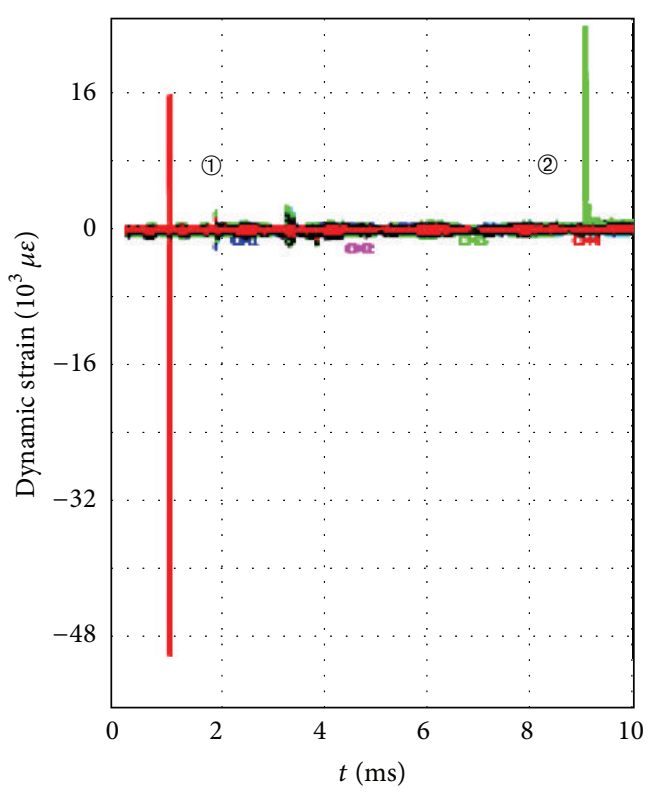

(b)

Figure 3: Measured curves of the blasting wave and the reflected wave from the free face.

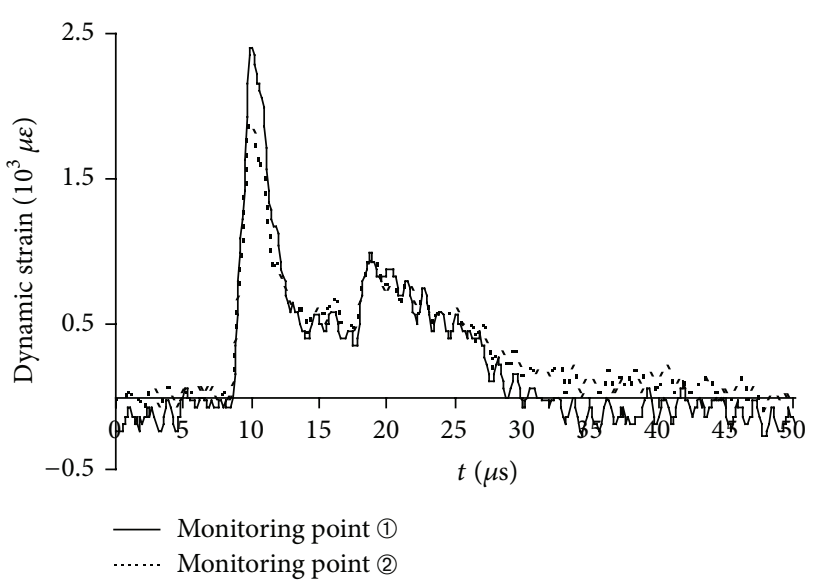

FIGURE 4: Measured curves of the reflected wave from the free face.

decreases and even disappears. If the loading and unloading properties of the whole blasting are considered, different results will be generated. If the unloading and the resilience of the shock wave are the ones measured in the zone, the zone should have the characteristics of the stress wave zone in the blasting wave division. However, as presented in numerous tests, the phenomenon of decreasing and disappearing exists in the second zone during the contacting blasting.

If the gas expansion causes a shock wave in the first zone, the effect of the wave front and the reaction zone must be ignored, a result which does not match detonation theory. Furthermore, no reference has ever recorded that the peak value of the gas expansion can reach more than $1 \mathrm{GPa}$. If the blasting cavity resilience and the second gas expansion occur in the second zone, then the time when the gas-expanding loading affects the blasting cavity should be determined.
However, only the loading of the blasting wave front and the reaction zone can affect the first zone, and there is no time for the explosion gas to expand and load in the first zone.

Therefore, based on ZND detonation theory, the mechanism of rock blasting, and the evidence of the blasting wave division in the test results, the shock wave zone in concrete results from the sudden jump in detonation pressure at the wave front compressive zone. Furthermore, the gasexpanding zone is caused by the amorphous isentropic expansion and flow of the detonation product after the C-J plane, and the mechanism in the stress wave zone in concrete is very complex. An in-depth discussion concerning the waves disappearing after the shock front of brittle materials, such as K9 glass, under high-speed loading was carried out by Zhao et al. [18]. After the blasting test, four cracks from the borehole center toward the minimum burden appear at the model surface (Figure 6).

The mechanical properties of materials are related to the strain rate and are different from the static mechanical properties. In the blasting wave, the function of the energy for concrete deformation is [19]

$$
E=F\left(E_{e}, E_{p}, E_{\Omega}, E_{x}\right),
$$

where $E_{e}$ is the elastic potential energy, $E_{p}$ is the plastic potential energy, $E_{\Omega}$ is the surface energy of newly formed surfaces, and $E_{x}$ includes the other kinds of energy.

In this test, assuming that $E_{x}=0$, then the process of energy transfer is as follows: (1) the phase of shock wave ascending: the concrete obtains energy $E_{\mathrm{sh}}\left(E_{\mathrm{sh}}=E_{e}+E_{p}\right)$ from the shock wave. If the energy $E_{\text {sh }}$ is higher than the threshold value $E_{\text {cri }}$ of crack development, new surface cracks will be formed; when $E_{\mathrm{sh}}<E_{\mathrm{cri}}$, no surface cracks appear and only the amount of microcracks in the concrete increases; 
TABLE 1: Peak values and calculations in each region.

\begin{tabular}{|c|c|c|c|c|c|}
\hline & t number & Shock wave zone & Stress wave zone & Gas-expanding zone & Total \\
\hline \multirow{8}{*}{ (1) } & t/us & $2.4-5.6 / 3.3$ & $5.6-8.6 / 3$ & $8.6-24.7 / 16.1$ & 22.4 \\
\hline & $f / \mathrm{KHz}$ & $0-829$ & $0-743$ & $0-514$ & $\times$ \\
\hline & $\sigma_{\max } / \mathrm{MPa}$ & -1650 & 159.72 & -65.01 & $\times$ \\
\hline & $\dot{\varepsilon}_{\max } / 10^{4} \mathrm{~s}^{-1}$ & $-7.58 / 10.5$ & $1.43 /-1.14$ & Approximate static load & $x$ \\
\hline & $u_{\max } / \mathrm{m} \cdot \mathrm{s}^{-1}$ & $-151.6 / 210$ & $28.5 /-22.7$ & Approximate static state & $\times$ \\
\hline & $a_{\max } / \mathrm{m} \cdot \mathrm{s}^{-2}$ & $612 /-505$ & $125 /-142$ & 0 & $\times$ \\
\hline & $E_{i}$ & 0.0157 & 0.0016 & 0.0054 & 0.0231 \\
\hline & $\eta_{i} / \%$ & 68.0 & 6.9 & 23.4 & 98.3 \\
\hline \multirow{8}{*}{ (2) } & $t / \mathrm{us}$ & $2.4-5.6 / 3.3$ & $5.6-8.6 / 3$ & $8.6-24.7 / 16.1$ & 22.4 \\
\hline & $f / \mathrm{KHz}$ & $0-1000$ & $0-686$ & $0-227$ & $\times$ \\
\hline & $\sigma_{\max } / \mathrm{MPa}$ & -1518 & 101.31 & 25.476 & $\times$ \\
\hline & $\dot{\varepsilon}_{\max } / 10^{4} \mathrm{~s}^{-1}$ & $-7.52 / 8.9$ & $1.24 /-1.12$ & Approximate static load & $x$ \\
\hline & $u_{\max } / \mathrm{m} \cdot \mathrm{s}^{-1}$ & $-148 / 178$ & $24.8 /-19.2$ & Approximate static state & $x$ \\
\hline & $a_{\max } / \mathrm{m} \cdot \mathrm{s}^{-2}$ & $608 /-413.5$ & $87 /-137.5$ & 0 & $x$ \\
\hline & $E_{i}$ & 0.0106 & 0.0012 & 0.0016 & 0.0137 \\
\hline & $\eta_{i} / \%$ & 77.3 & 8.8 & 11.7 & 97.8 \\
\hline
\end{tabular}

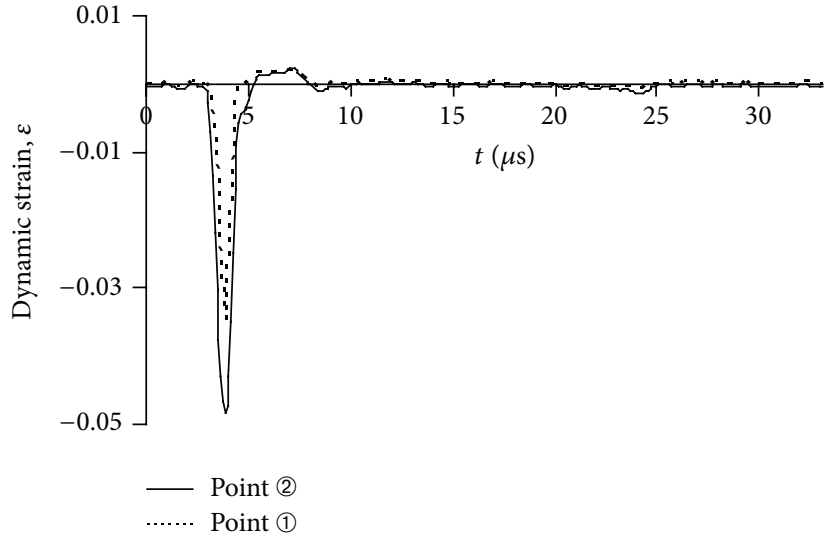

(a)

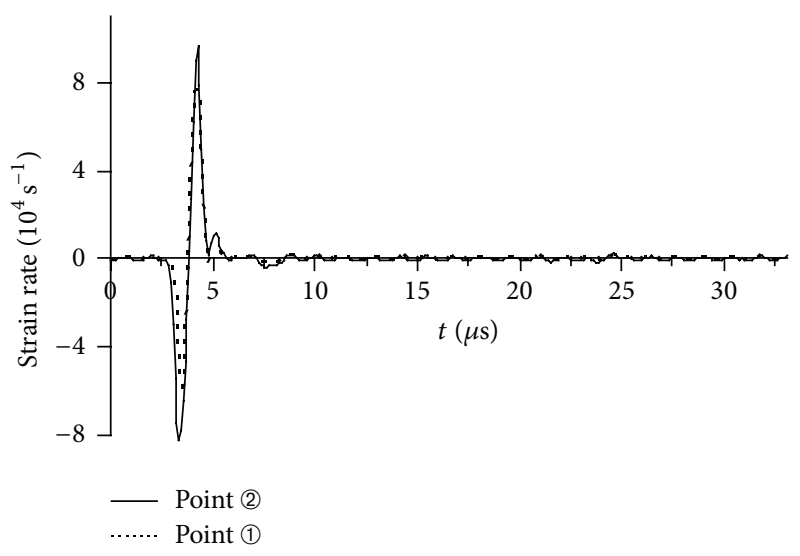

(b)

FIGURE 5: Measured curve of contacting blasting waveform.

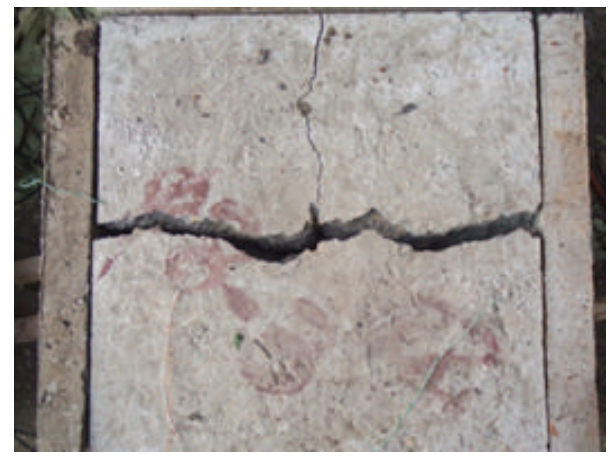

FIgURE 6: Distribution of blasting cracks in the $\phi 8 \mathrm{~mm}$ model.

(2) the phase of shock wave unloading: two types of energy, namely, deformation reversion energy $E_{r}$ and wasted energy $E_{d}$, are released from $E_{\mathrm{sh}}$, between which $E_{d}$ is the main cause of formation of new surface cracks or microcracks; (3) the phase of the effects of stress wave and explosion gas: the types of energy the concrete gets from the stress wave and the explosion gas are $E_{\mathrm{st}}$ and $E_{g}$, respectively. If $E_{\mathrm{st}}>E_{\mathrm{cri}}$ or $E_{g}>E_{\text {cri }}$, then new surface cracks will form; otherwise, only the existing cracks caused by $E_{\mathrm{sh}}$ can develop further. Based on the calculations in Table 1, $E_{\text {st }}$ and $E_{g}$ are much smaller than $E_{\text {sh }}$, and the effect of $E_{\mathrm{sh}}$ is mainly reflected in the crack development.

\section{Conclusions}

(1) In the test of blasting wave in concrete with coupling charge, the following may be concluded in terms of space: the monitoring area, which is $20 R_{0}-40 R_{0}$ away from the blasting center, is subjected to the combined effects of blasting shock wave, stress wave, and gas expansion. In terms of time, the three waves affect 
the points. Concrete damage results from a complex process under shock compression and tension of the blasting shock wave, as well as the blasting stress wave at a strain rate of more than $10^{4} / \mathrm{s}$, and the effect of gas expansion at an approximate static state.

(2) The relationship between the effected process of blasting wave and the concrete damage is quantitatively analyzed in terms of energy. If the shock wave energy is greater than the releasing rate of energy for crack development, new surface cracks appear; otherwise, only microcracks appear, and the energy of the stress wave and that of the gas expansion are much smaller than the shock wave energy whose effect is mainly reflected in the crack development.

\section{Conflict of Interests}

The authors declare that there is no conflict of interests regarding the publication of this paper.

\section{Acknowledgment}

The research project was supported by National Natural Science Foundation of China (41202057).

\section{References}

[1] J. Latham, A. Munjiza, and P. Lu, "Components in an understanding of rock blasting," in Proceedings of the 6th International Symposium on Rock Fragmentation by Blasting, pp. 173-182, Johannesburg, South Africa, 1999.

[2] J. A. Sanchidrián, P. Segarra, and L. M. López, "Energy components in rock blasting," International Journal of Rock Mechanics and Mining Sciences, vol. 44, no. 1, pp. 130-147, 2007.

[3] H. Lin, Z. Xiong, T. Liu, R. Cao, and P. Cao, "Numerical simulations of the effect of bolt inclination on the shear strength of rock joints," International Journal of Rock Mechanics and Mining Sciences, vol. 66, pp. 49-56, 2014.

[4] O. S. Dinc, H. Sonmez, C. Tunusluoglu, and K. E. Kasapoglu, "A new general empirical approach for the prediction of rock mass strengths of soft to hard rock masses," International Journal of Rock Mechanics and Mining Sciences, vol. 48, no. 4, pp. 650-665, 2011.

[5] J. González-Cao, F. Varas, F. G. Bastante, and L. R. Alejano, "Ground reaction curves for circular excavations in nonhomogeneous, axisymmetric strain-softening rock masses," Journal of Rock Mechanics and Geotechnical Engineering, vol. 5, no. 6, pp. 431-442, 2013.

[6] I.-S. Ha, "Estimation of shear wave velocity of earth dam materials using artificial blasting test," Soil Dynamics and Earthquake Engineering, vol. 55, pp. 120-129, 2013.

[7] A. K. Chakraborty, J. L. Jethwa, and A. G. Paithankar, "Effects of joint orientation and rock mass quality on tunnel blasting," Engineering Geology, vol. 37, no. 3-4, pp. 247-262, 1994.

[8] R. P. Dhakal and T.-C. Pan, "Response characteristics of structures subjected to blasting-induced ground motion," International Journal of Impact Engineering, vol. 28, no. 8, pp. 813-828, 2003.

[9] A. Mortazavi and P. D. Katsabanis, "Modelling burden size and strata dip effects on the surface blasting process," International
Journal of Rock Mechanics and Mining Sciences, vol. 38, no. 4, pp. 481-498, 2001.

[10] S.-G. Wen, Y.-Q. Gong, S.-N. Dong, S.-Y. Wang, and S.-Y. Tang, "Application of wide range pressure gauge to DDT experiments on energetic materials," Chinese Journal of Energetic Materials, vol. 15, no. 2, pp. 165-168, 2007.

[11] S. Bhandari, "On the role of stress waves and quasi-static gas pressure in rock fragmentation by blasting," Acta Astronautica, vol. 6, no. 3-4, pp. 365-383, 1979.

[12] J. C. Jhanwar, J. L. Jethwa, and A. H. Reddy, "Influence of airdeck blasting on fragmentation in jointed rocks in an open-pit manganese mine," Engineering Geology, vol. 57, no. 1-2, pp. 1329, 2000.

[13] M. Zbikowski, D. Makarov, and V. Molkov, "LES model of large scale hydrogen-air planar detonations: verification by the ZND theory," International Journal of Hydrogen Energy, vol. 33, no. 18, pp. 4884-4892, 2008.

[14] G. Lyng and K. Zumbrun, "A stability index for detonation waves in Majda's model for reacting flow," Physica D: Nonlinear Phenomena, vol. 194, no. 1-2, pp. 1-29, 2004.

[15] G. D. Roy, S. M. Frolov, A. A. Borisov, and D. W. Netzer, "Pulse detonation propulsion: challenges, current status, and future perspective," Progress in Energy and Combustion Science, vol. 30, no. 6, pp. 545-672, 2004.

[16] M. Ramulu, A. K. Chakraborty, and T. G. Sitharam, "Damage assessment of basaltic rock mass due to repeated blasting in a railway tunnelling project-a case study," Tunnelling and Underground Space Technology, vol. 24, no. 2, pp. 208-221, 2009.

[17] J. Yang, Q. K. Jin, and F. L. Huang, Theoretical Models of Rock Blasting and Numerical Calculation, Science Press, Beijing, China, 1999.

[18] J.-H. Zhao, Z.-P. Duan, X.-X. Tan et al., "Investigations of failure waves in K9 glass using shadowgraph," Explosion and Shock Waves, vol. 21, no. 2, pp. 150-156, 2001.

[19] H. Schuler, C. Mayrhofer, and K. Thoma, "Spall experiments for the measurement of the tensile strength and fracture energy of concrete at high strain rates," International Journal of Impact Engineering, vol. 32, no. 10, pp. 1635-1650, 2006. 

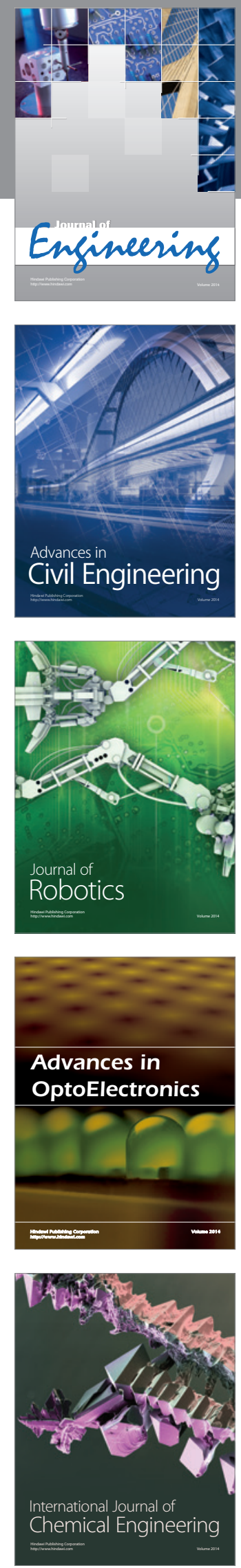

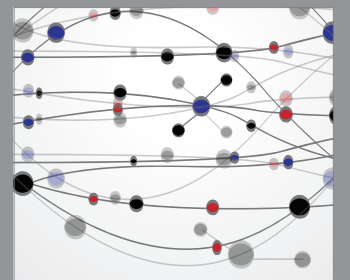

The Scientific World Journal
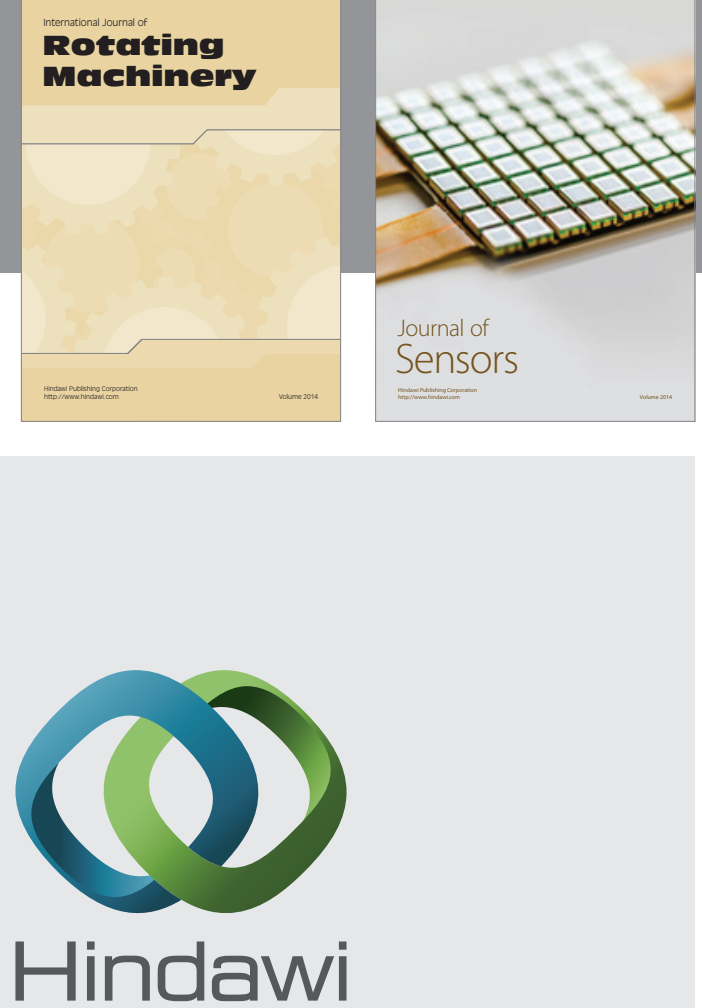

Submit your manuscripts at http://www.hindawi.com
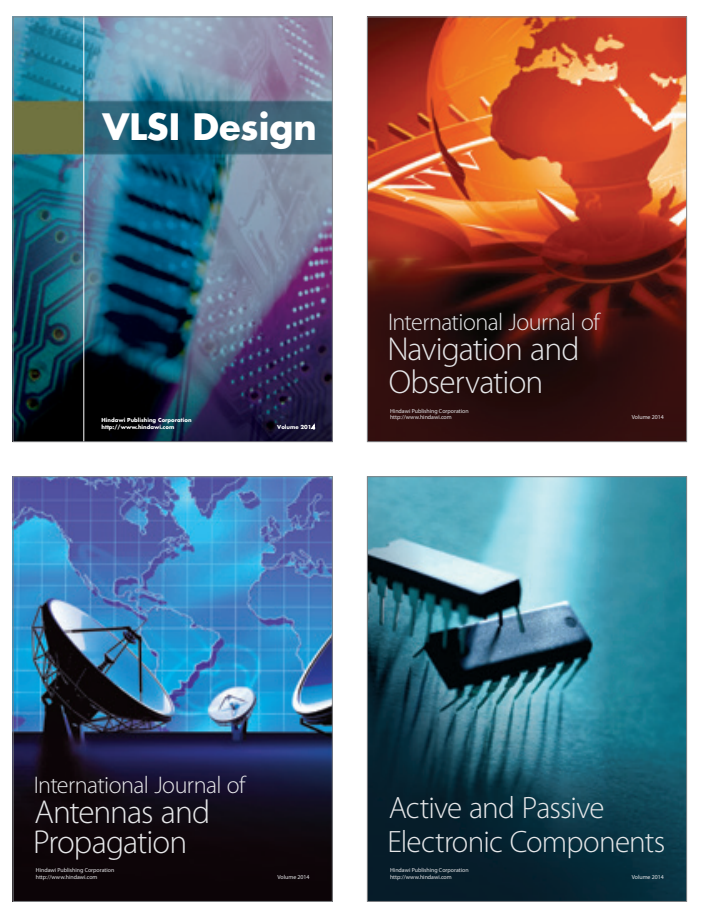
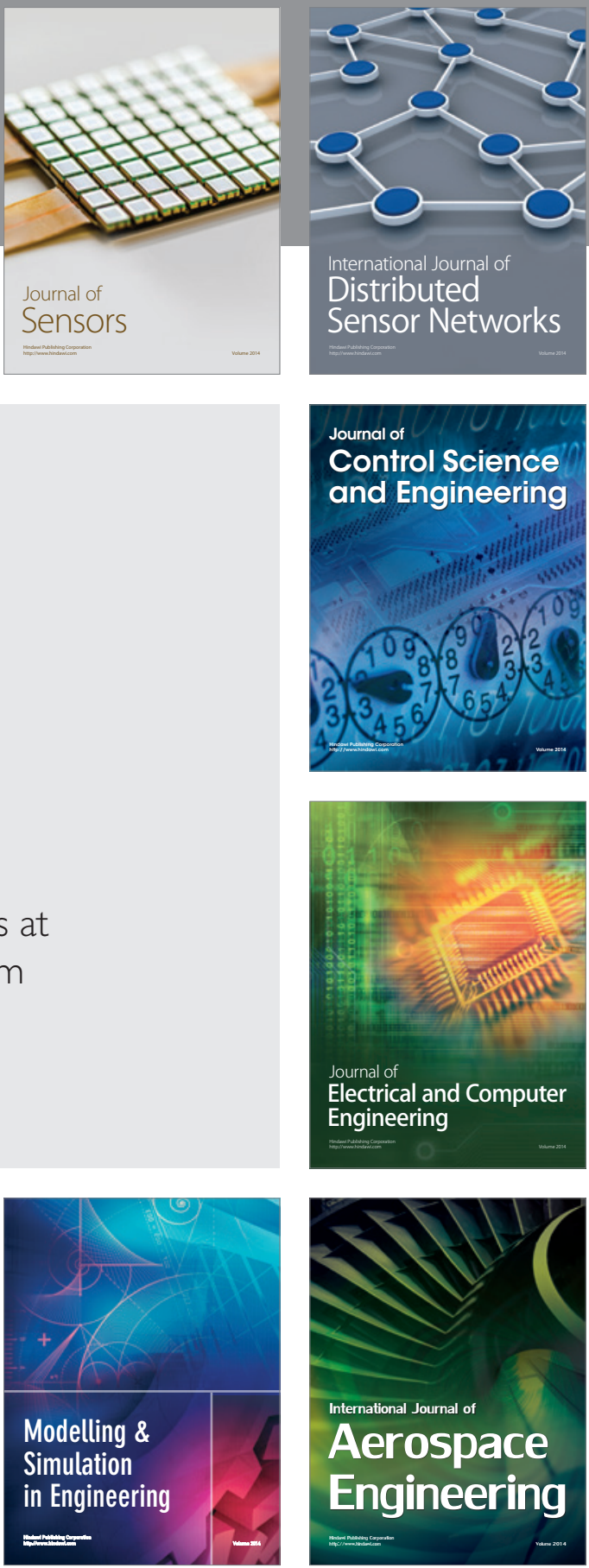

Journal of

Control Science

and Engineering
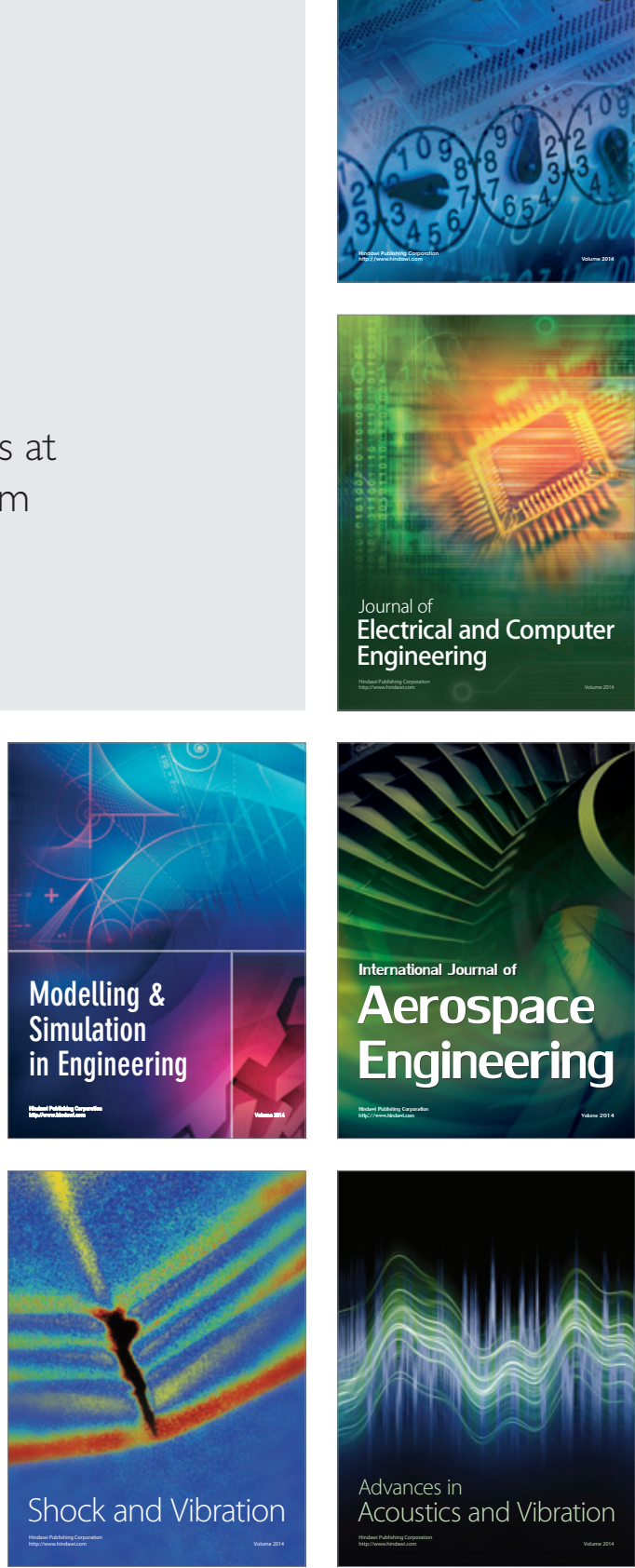